\title{
Stabilization of Axon Branch Dynamics by Synaptic Maturation
}

\author{
Edward S. Ruthazer, ${ }^{1,2}$ Jianli Li, ${ }^{1}$ and Hollis T. Cline ${ }^{1}$ \\ ${ }^{1}$ Cold Spring Harbor Laboratory, Cold Spring Harbor, New York 11724-0100, and ${ }^{2}$ Montreal Neurological Institute, Department of Neurology and \\ Neurosurgery, McGill University, Montréal, Quebec, Canada H3A 2B4
}

The developmental refinement of topographic projections in the brain is reflected in the dynamic sculpting of axonal arbors that takes place as connections between CNS structures form and mature. To examine the role of synaptogenesis and synaptic maturation in the structural development of axonal projections during the formation of the topographic retinotectal projection, we coexpressed cytosolic fluorescent protein (FP) and FP-tagged synaptophysin (SYP) in small numbers of retinal ganglion cells in living albino Xenopus laevis tadpoles to reveal the distribution and dynamics of presynaptic sites within labeled retinotectal axons. Two-photon time-lapse observations followed by quantitative analysis of tagged SYP levels at individual synapses demonstrated the time course of synaptogenesis: increases in presynaptic punctum intensity are detectable within minutes of punctum emergence and continue over many hours. Puncta lifetimes correlate with their intensities. Furthermore, we found that axon arbor dynamics are affected by synaptic contacts. Axon branches retract past faintly labeled puncta but are locally stabilized at intensely labeled SYP puncta. Visual stimulation for $4 \mathrm{~h}$ enhanced the stability of the arbor at intense presynaptic puncta while concurrently inducing the retraction of exploratory branches with only faintly labeled or no synaptic sites.

Key words: in vivo imaging; synapse maturation; axon arbor plasticity; retinotectal; Xenopus; synaptogenesis

\section{Introduction}

Patterned neural activity and synaptic transmission guide the remodeling of axonal and dendritic processes in the developing CNS by regulating the addition, stabilization, and elimination of branches as arbors grow (O'Rourke et al., 1994; Rajan et al., 1999; Sin et al., 2002; Hua et al., 2005). In the retinotectal projection, the dynamic rearrangement of axonal branches actively refines and maintains the retinotopic map (Ruthazer and Cline, 2004; McLaughlin and O'Leary, 2005). This is particularly evident in fish and tadpoles, where the eyes and the brain both undergo protracted growth involving the addition of new neurons along different axes (Easter and Stuermer, 1984; Reh and ConstantinePaton, 1984). Retinal topography is maintained by the continual shifting of retinal ganglion cell (RGC) axon arbors within the optic tectum. Axon remodeling involves the selective stabilization of some branches, whereas others are eliminated, under the control of activity-dependent Hebbian mechanisms involving retrograde signaling downstream of activation of postsynaptic tectal NMDA receptors, which effectively function as correlation detectors (Cline and Constantine-Paton, 1989; Schmidt and Buzzard, 1993; Schmidt et al., 2000; Ruthazer et al., 2003).

Beyond its fundamental role in synaptic transmission, the

Received Jan. 7, 2006; revised Feb. 12, 2006; accepted Feb. 14, 2006.

This work was supported by awards from the National Alliance for Research on Schizophrenia and Depression (E.S.R.) and the National Eye Institute (H.T.C.) and by the National Institutes of Health Director's Pioneer Award (H.T.C.). We acknowledge the expert technical assistance of Kimberly Bronson, Chih-ming Chen, and Stephen Hearn. We thank the members of the Cline laboratory for illuminating discussions. We are grateful to Dr. Jane Sullivan for the gift of SYP-GFP plasmid, Dr. Cyrille Alexandre for the HRP plasmid, and Dr. Benjamin Glick for the dsRED-MST.

Correspondence should be addressed to Hollis Cline, Cold Spring Harbor Laboratory, 1 Bungtown Road, Cold Spring Harbor, NY 11724. E-mail: cline@cshl.org.

D01:10.1523/JNEUROSCI.0069-06.2006

Copyright $\odot 2006$ Society for Neuroscience $\quad$ 0270-6474/06/263594-10\$15.00/0 synapse is a dynamic aggregate of scaffolding and signaling molecules that changes as the synapse matures (Vaughn, 1989; McGee and Bredt, 2003; Ziv and Garner, 2004). Maturation of the presynaptic terminal is evident both functionally as an increase in the reliability of transmitter release and morphologically as an increase in the number of presynaptic vesicles (Sudhof, 2000). Several studies have used time-lapse imaging of neurons expressing green fluorescent protein (GFP)-tagged presynaptic proteins to observe the development of synapses in cultured neurons (Ahmari et al., 2000; Shapira et al., 2003) or in Xenopus retinotectal axons in vivo (Alsina et al., 2001; Hu et al., 2005). These in vivo studies found that vesicle-associated membrane protein (VAMP)-GFP puncta constituted the principal sites of a new branch addition and that branches that failed to form VAMPGFP puncta were more likely to be eliminated. Notably, a similar phenomenon occurs in motor neuron axon development in $\mathrm{Xe}$ nopus (Javaherian and Cline, 2005) and zebrafish (Panzer et al., 2006). These observations suggest a central role for the synapse in axon arbor remodeling and raise the intriguing possibility that as synapses mature and strengthen, they may serve double duty as structural sites for axon branch stabilization.

We therefore asked whether maturing synapses come to act as stabilization points along dynamic axonal branches. We used a nonbiased quantitative approach to identify and measure changes in presynaptic vesicle accumulation over time at synaptic sites in RGC axons in living Xenopus tadpoles. We then related these changes in synapse maturation to structural dynamics of axons. We find that sites of dense presynaptic vesicle accumulation correspond to the locations where axon branches are stabilized against retraction. Furthermore, visual stimulation enhances the stability of the axon arbor at synapses that have accumulated many vesicles while destabilizing the axon arbor at 
immature synapses, leading to refinement of arbor structure. These data reveal a direct correlation between synaptic maturation and the local stability of axon branches and thus indicate a role for synaptic development in topographic map refinement.

\section{Materials and Methods}

RGC transfection in tadpoles. RGCs were transfected by one of two methods. For transfection in early retinal development, stage 23 (Nieuwkoop and Faber, 1956) albino Xenopus tadpoles were dejellied by immersion in $2 \%$ cysteine for $\sim 5 \mathrm{~min}$, after which they were thoroughly washed in rearing solution ( $0.1 \%$ Steinberg's). Under a dissecting microscope, a small volume of a lipofection mixture, consisting of $3 \mu \mathrm{l}$ of plasmid DNA $[\sim 1 \mu \mathrm{g} / \mu \mathrm{l}$ in water with 1:3 ratio of synaptophysin (SYP)-GFP and Discosoma red fluorescent protein (dsRED)-MST plasmids] in $9 \mu \mathrm{l}$ of $N$-[1-(2,3-dioleoyloxy)propyl]-N,N,N-trimethylammonium methylsulfate lipofectant (DOTAP; Roche Products, Welwyn Garden City, UK), was pressure injected into the eye primordium of the tadpole from a finely pulled glass micropipette mounted on a Picospritzer II (General Valve, Fairfield, NJ). A small amount of fast green dye was added to the mixture to help visualize the delivery of solution.

For late stage transfection, stage 44-48 tadpoles were briefly anesthetized in $0.02 \%$ MS-222 (Sigma, St. Louis, MO) and placed on a moist kimwipe under a dissecting microscope. A small volume, enough to visualize but not distend the eye, of plasmid DNA solution $[\sim 1 \mu \mathrm{g} / \mu \mathrm{l}$ in water; 1:3 ratio of SYP conjugated to enhanced cyan fluorescent protein (ECFP) to enhanced yellow fluorescent protein (EYFP) from Clontech, Mountain View, CA], colored with fast green dye, was pressure injected into the vitreous humor between the lens and the retina. Immediately afterward, a pair of custom-made platinum plate electrodes was positioned to span the eye with the anode closest to the region of the eye to be transfected. Approximately six pulses were delivered across the electrodes using an electrical stimulator (Grass SD9; Grass Instruments, Quincy, MA) set to 30-80 V intensity and $1.6 \mathrm{~ms}$ duration. To produce an exponential decay waveform, a $3 \mu \mathrm{F}$ capacitor was placed in parallel with the electrodes.

The GFP/dsRED-MST combination was brighter and less sensitive to photobleaching, whereas the superior spectral distinction of the CFP/ YFP combination permitted imaging SYP-CFP without bleedthrough of emission fluorescence from the YFP. We also examined expression patterns of a different synaptic marker, VAMP-GFP. However, in our study, this construct produced a more continuous labeling along the length of the axon and thus would have required subtraction and thresholding for punctum identification (data not shown).

Immunohistochemistry. Tadpoles with SYP-GFP-expressing RGC axons were fixed in 3.5\% paraformaldehyde and $0.1 \%$ glutaraldehyde for 1-2 $\mathrm{h}$. The brains were dissected and then embedded in albumin-gelatin mixture. The tissue blocks were cut into $30 \mu \mathrm{m}$ sections on a vibratome (Vibratome 1500; Technical Products International, St. Louis, MO). After preincubation in $10 \%$ normal goat serum in $0.1 \mathrm{M}$ Tris- $\mathrm{HCl}$ buffer for $30 \mathrm{~min}$, the sections were then incubated at $4^{\circ} \mathrm{C}$ overnight with antipostsynaptic density-95 (PSD-95) antibody (rabbit polyclonal, 1:500 dilution; Zymed, San Francisco, CA). Alexa568 anti-rabbit IgG (1:1000 dilution; Invitrogen, Eugene, OR) was used as the secondary antibody. Finally, sections were coverslipped and imaged at $0.5 \mu \mathrm{m}$ intervals for GFP and PSD-95 immunostaining with a laser-scanning confocal microscope. Juxtaposition was defined as overlapping of an SYP-GFP punctum and a PSD-95 punctum in at least two consecutive optical sections. Only those PSD-95 puncta with a diameter $>0.5 \mu \mathrm{m}$ were counted.

Electron microscopy. Tadpoles with biotinylated dextran amine (BDA) or horseradish peroxidase (HRP)-SYP-GFP-expressing RGC axons were fixed in $1 \%$ paraformaldehyde and $1.5 \%$ glutaraldehyde for $1-2 \mathrm{~h}$. The brains were sectioned at $50 \mu \mathrm{m}$ on a vibrotome. After cryoprotection in $15 \%$ DMSO and 2\% glycerol, the sections were freeze-thawed in a freezer at $-80^{\circ} \mathrm{C}$. Sections with $\mathrm{HRP}$ were enhanced using the tyramide signal amplification biotin system (PerkinElmer, Emeryville, CA) and reacted with nickel-intensified diaminobenzidine (DAB; Sigma) for 5-10 min. Sections with BDA-labeled axons were preincubated in 10\% normal goat serum in $0.1 \mathrm{M}$ Tris- $\mathrm{HCl}$ buffer for $30 \mathrm{~min}$ and then incubated overnight at $4^{\circ} \mathrm{C}$ in a 1:50 dilution of avidin and biotinylated HRP (Vector Laboratories, Burlingame, CA) in Tris- $\mathrm{HCl}$ buffer. After buffer washes, sections were reacted with nickel-intensified DAB for 5-10 min. Selected sections were postfixed in $2 \%$ osmium tetroxide, dehydrated in an acetone series, and flat-embedded in Durcupan resin (Electron Microscopy Sciences, Fort Washington, PA) between two sheets of Aclar plastic (Ladd Research, Williston, VT). The areas with optic tectum were mounted on blocks, and ultrathin sections (70-80 nm; silver-gray interference color) were cut using a diamond knife (Diatome, Biel, Switzerland). All ultrathin sections were collected on formvar-coated nickel slot grids before examination with a Hitachi (Tokyo, Japan) 7500 electron microscope. $\mathrm{NIH}$ ImageJ was used for analysis of size distribution of terminals and postsynaptic profiles.

Two-photon imaging. Tadpoles at stage 48 were screened for isolated single RGC axon arbors in the optic tectum under epifluorescent illumination using a $20 \times$ objective, taking care to minimize time of exposure to excitation light. For imaging, tadpoles were anesthetized in $0.02 \% \mathrm{MS}$ 222 solution and immobilized under a glass slide in a Sylgard chamber, shaped to closely fit a tadpole's body. Images were collected at regular intervals, ranging from every $10 \mathrm{~min}$ to every $4 \mathrm{~h}$. Two-photon $\mathrm{z}$-series were collected using a custom modified Olympus (Tokyo, Japan) FV300 scan head, focus motor, and software. Excitation light at $910 \mathrm{~nm}$ was generated either by a Tsunami femtosecond pulsed infrared laser pumped with a Millenia V diode-pumped laser (Spectra Physics, Fremont, CA) or by a Mira-pulsed infrared laser pumped with a Verdi diode laser (Coherent, Kitchener, Ontario, Canada). Fluorescence emission was detected using a pair of R3896 photomultiplier tubes (Hamamatsu, Shizuoka, Japan), mounted in the reflected beam path of a 700LP dichroic mirror. To simultaneously image ECFP and EYFP fluorescence emission, a 510DCLP beamsplitter was used with HQ480/40 and HQ540/40 filters. To image GFP and dsRED-MST, a 565DCLPXR beamsplitter was used with HQ525/50 and HQ607/45 filters (Chroma Technology, Brattleboro, VT). Signals were processed using custom built transimpedance amplifiers and digitally acquired at $512 \times 512$ pixel resolution with a pixel depth of 12 bits per channel. Laser intensity was monitored using a photodiode and manually maintained at constant intensity. Two-photon z-series were collected at $1 \mu \mathrm{m}$ intervals using a $40 \times$ water immersion objective (numerical aperture, 0.8 ; Olympus) at $3 \times$ scan zoom.

Calculation of punctum intensity fluctuation over time. The measured intensities of individual SYP puncta on the main axon shaft can wax and wane by $21.1 \pm 1.9 \%$ per $10 \mathrm{~min}$ imaging interval (see Fig. $2 e$ ). Optical sections were collected at $1 \mu \mathrm{m}$ intervals, which assured that no puncta or fine branches were missed, but did not extensively oversample the specimen to minimize exposure to harmful excitation light. Therefore, the fluctuation in punctum intensity reflects both real changes in SYP accumulation as well as artifact because of variability in centering of optical sections collected at different time points. The contribution of axial centering to the fluctuation should be proportional across all punctum intensities, whereas changes resulting from vesicle accumulation or loss should be small and additive. Consequently, fluctuations of bright puncta mainly reflect optical sectioning artifact, whereas fluctuations of faint puncta could reflect both real and artifactual changes. We examined puncta along the portion of the axon shaft shown in Figure $2 c$ over a $1 \mathrm{~h}$ period and found that image-to-image fluctuation of the brightest puncta $(>100$ intensity; $n=15)$ was $12.2 \pm 3.0 \%$ of average intensity, whereas the fainter puncta ( $<100$ intensity; $n=73$ ) fluctuated by $23.0 \pm$ $2.2 \%$. The optical sectioning artifact therefore introduces $\sim 12 \%$ noise to the intensity measurements. Thus, the synaptic vesicle content in immature synapses can fluctuate over the time course of minutes.

Visual stimulation. Freely swimming tadpoles were placed individually in wells of a 12 -well dish in a black Plexiglas chamber with a $3 \times 4$ panel of green light-emitting diodes (LEDs; 567 nm; Allied Electronics, Fort Worth, TX) on the top of the chamber (Sin et al., 2002). After collection of a $0 \mathrm{~h}$ starting image, the tadpoles were placed in the dark chamber for $4 \mathrm{~h}$ without activating the LEDs. A second image was then collected, and the tadpoles were returned to the chamber for $4 \mathrm{~h}$ of visual stimulation. During this period, each row of LEDs turned on and remained on for $1 \mathrm{~s}$ 
at a frequency of $0.2 \mathrm{~Hz}$. The rows turned on and off sequentially to create a simulated motion stimulus, with $1 \mathrm{~s}$ of darkness between each cycle. A final image was collected after stimulation.

Axon reconstruction and punctum analysis. Individual axonal arbors were manually reconstructed in three dimensions from two-photon z-series stacks using custom Object-Image macros. Using the SYP channel image, individual axon branches were digitally linearized by arranging into a straight line each of the individual line segments that make up each reconstructed axon branch. For automated identification of SYP puncta, the fluorescence intensity (six pixel, $1.5 \mu \mathrm{m}$, wide window) along the entire length of each axon branch was measured. The criteria for puncta were that the fluorescence intensity must undergo at least a $20 \%$ increase within two consecutive pixels $(0.5 \mu \mathrm{m})$ followed by a $20 \%$ decrease in intensity units within two consecutive pixels. In addition, the points of rising and falling fluorescent intensity must fall within eight pixels $(2 \mu \mathrm{m})$ of each other. For this analysis, image lookup tables were scaled so that 10 or fewer pixels in the entire stack had saturated intensities $(\geq 255)$, and background had a value of 1 . The software collected information about punctum brightness (mean intensity within a $7 \times 6$ pixel window) and relative location for later analysis. For normalization of punctum intensities, the distribution of intensities of all puncta was linearly scaled to set the 80th percentile brightest punctum equal to a relative intensity unit value of 80 for each axon. SEM is given throughout the text and figures.

\section{Results}

Synaptophysin-GFP labels presynaptic sites in vivo

Synaptic sites in RGC axons were visualized in vivo by transfecting individual cells with a plasmid encoding a fusion of enhanced GFP to the C terminus of murine SYP, a major integral membrane protein of synaptic vesicles. We chose to use tagged SYP to visualize synaptic development in vivo based on several properties of the protein. First, because SYP is specifically targeted to synaptic vesicles, it is an ideal indicator of changes in vesicle number at the synapse. Second, it consistently produced a clear punctate pattern of labeling in axon terminals, with little or no labeling along the intervening axonal length. The discrete, punctate pattern of SYPGFP fluorescence in RGC axon arbors was mainly localized to varicosities distributed regularly throughout the axon arbor within the optic tectum (Fig. 1a). Axon arbor morphology and synaptic localization were visualized simultaneously by cotransfecting GFPtagged SYP (SYP-GFP) together with a rapidly maturing variant of red fluorescent protein (dsRED-MST) (Bevis and Glick, 2002) or enhanced cyan fluorescent protein-tagged SYP (SYP-CFP) with cytosol filling enhanced YFP (see Materials and Methods). The distribution of tagged SYP fluorescence did not simply reflect volume differences along the axon, because large nonsynaptic structures with bright dsRED fluorescence, such as growth cones and axon shafts in the optic tract, had barely detectable SYP-GFP fluorescence.

The punctate distribution of tagged SYP represents the accumulation of synaptic vesicles at presynaptic terminals. Immunostaining for the postsynaptic marker PSD-95 in histological sec-
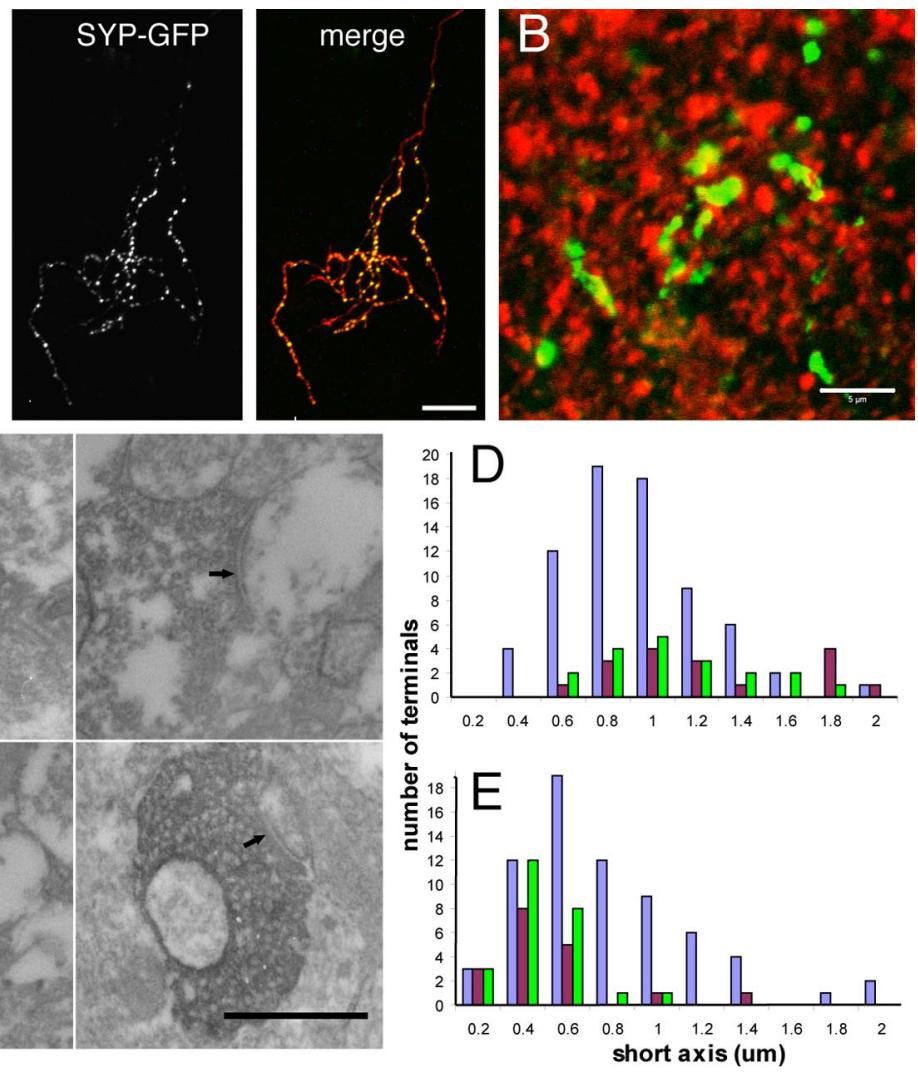

Figure 1. SYP-GFP labels presynaptic sites in vivo. $\boldsymbol{A}$, Coexpression of SYP-GFP with dsRED-MST or SYP-CFP with EYFP in RGCS reveals discrete SYP puncta concentrated in the axon arbor in the optic tectum. These colocalize with the varicosities normally roduct in synaptic vesicle membranes at morphologically identifiable synaptic sites (arrows). $D$. The distribution of presynaptic axons (brown). Both were larger than the presynaptic terminals of unlabeled (nonretinal) synapses (purple) in the optic tectum. $\boldsymbol{E}$ The contacted postsynaptic profiles were also identical for HRP-SYP-GFP-labeled RGC terminals and control biotin dex labeled RGC axons. Scale bars: $\boldsymbol{A}, 10 \mu \mathrm{m} ; \boldsymbol{B}, 5 \mu \mathrm{m} ; \boldsymbol{C}, 500 \mathrm{~nm}$

tions containing SYP-GFP-expressing axons reliably revealed the juxtaposition of SYP-GFP puncta to sites of PSD-95 immunoreactivity (Fig. 1b). To confirm this at the ultrastructural level, RGCs were transfected with a plasmid encoding HRP fused to the SYP-GFP construct (HRP-SYP-GFP). HRP-SYP-GFP produced an electron dense precipitate when reacted with $\mathrm{DAB}$, permitting visualization under the electron microscope. Ultrastructural analysis showed that 95\% (19 of 20) of labeled puncta were vesicle-bearing RGC synaptic terminals that contacted dendritic profiles with postsynaptic densities (Fig. 1c). It is noteworthy that all presynaptic vesicles in a labeled terminal appeared to be positive for the dark DAB reaction product, indicating that the majority of vesicles have incorporated the fusion protein. This suggests that changes in fluorescence intensity of the presynaptic puncta correspond to changes in presynaptic vesicle content at the synaptic terminal. Interestingly, in many cases, a single presynaptic cluster of vesicles exhibited synaptic contacts onto multiple postsynaptic partners (data not shown). The expression of exogenous synaptophysin did not alter the size distribution of either the presynaptic terminals of transfected axons (Fig. 1d) or their associated postsynaptic profiles (Fig. 1e) compared with those of control biotin-labeled RGC axons, suggesting that synaptic structure was not affected by expression of the tagged SYP. 


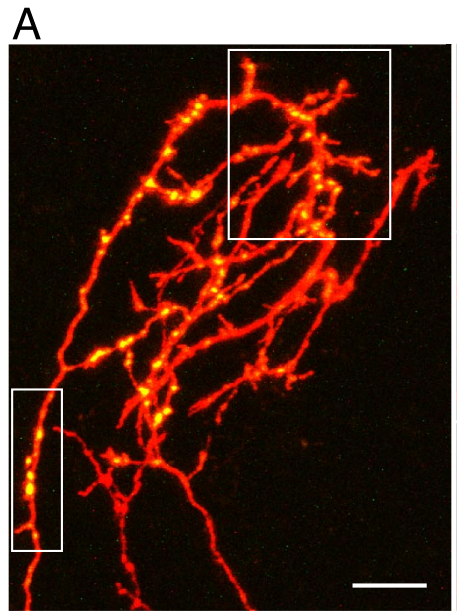

\section{B}

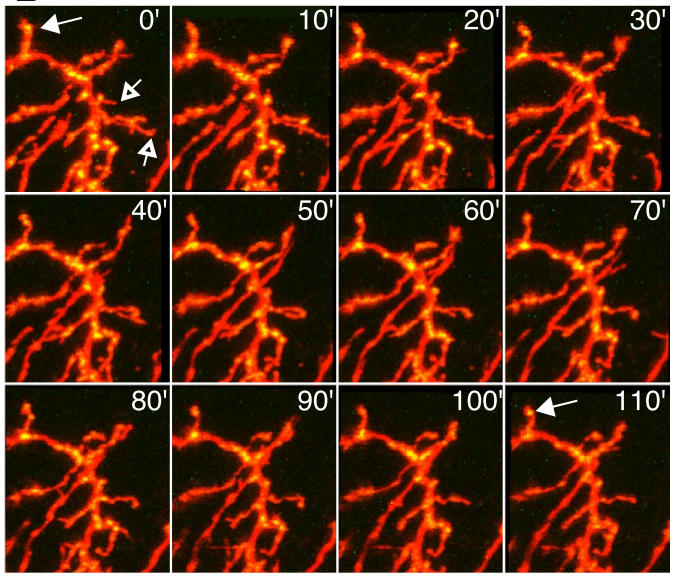

C
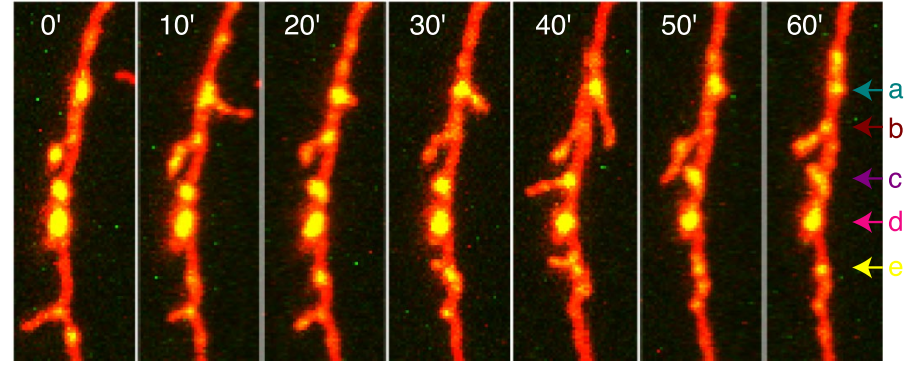

\section{$\mathrm{D}$}

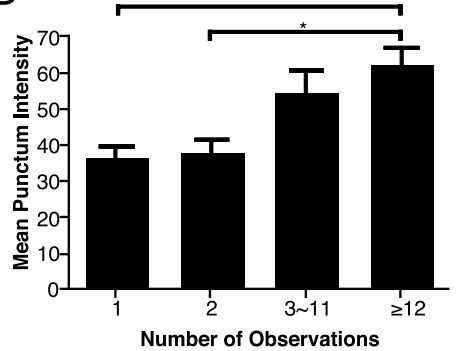

$\mathrm{E}$

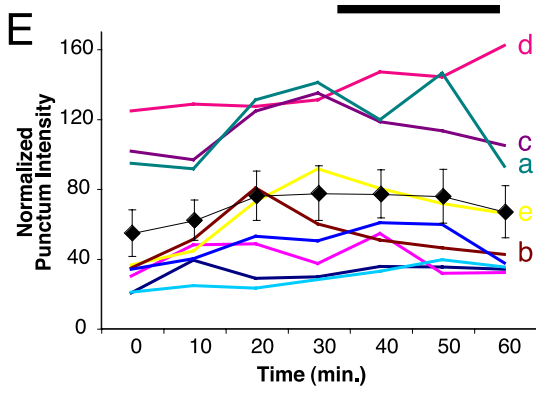

Figure 2. Rapid branch and synapse dynamics of SYP-CFP plus YFP-expressing RGC axons in vivo. A, A cluster of three RGC axon arbors elaborating in the optic tectum. Two are coexpressing SYP-CFP (green) and YFP (red). Boxed areas are shown as time-lapse series in $\boldsymbol{B}$ and $\boldsymbol{C}$. Images are projections of two-photon z-series collected at $10 \mathrm{~min}$ intervals. $\boldsymbol{B}$, Stable branches bear bright SYP puncta (solid arrows), whereas transient branches typically have faint or no puncta (open arrows). Most new branches emerge from sites with SYP puncta. C, Axon shaft segment adding and losing interstitial branches over $1 \mathrm{~h}$. D, Mean punctum intensity on individual branches correlates with branch lifetime. Transient branches lasting $<30$ min have fainter puncta than stable branches persisting $\geq 2 \mathrm{~h}$. $\boldsymbol{E}$, Fluctuation of the intensities of stable puncta from the axon segment that includes $C$. Mean intensity is shown as black diamonds. ${ }^{*} p<0.05,{ }^{* *} p<0.01$; Kruskal-Wallis with Dunn posttest. Scale bars, $10 \mu \mathrm{m}$.

\section{New branches emerge from SYP puncta}

RGC axon terminals in the optic tectum grow by a process of extensive interstitial branch addition and retraction along the entire axon arbor rather than by splitting of a leading growth cone (Kaethner and Stuermer, 1992; O’Rourke et al., 1994). Interstitial branches are typically small $(<5 \mu \mathrm{m})$, highly dynamic, and shortlived (Witte et al., 1996). A fraction of these interstitial branches will be maintained and serve as sites for further branch addition (Kaethner and Stuermer, 1992; O’Rourke et al., 1994; Witte et al., 1996; Alsina et al., 2001; Ruthazer et al., 2003; Hua et al., 2005). It has long been speculated that synaptic sites participate in this process, but this has not yet been directly demonstrated.

Previous studies in which developing axons were imaged at intervals of several hours or days suggested that axon branch sites are frequently located near synaptic puncta both in the central and peripheral nervous system (Alsina et al., 2001; Javaherian and Cline, 2005; Panzer et al., 2006). Consistent with these reports, the majority of side branches $(78 \%)$ that we observed had intense SYP puncta at their branch points on the parent axon. By collecting time-lapse two-photon z-series of axons coexpressing EYFP and SYP-CFP at $10 \mathrm{~min}$ intervals (Fig. 2), we observed that SYP puncta are the sites of origin of a large number of highly dynamic exploratory branches. These newly added branches extend from sites with bright puncta $81 \%$ of the time (38 of 47 new branches in $2 \mathrm{~h}$ ). We found no relationship between the relative intensities of puncta at branch bases and the stability of the branches that emerged from them.

\section{Intensity of SYP puncta correlates with} branch lifetimes

In contrast, branch stability was correlated with the relative intensity of synaptic puncta on the distal end of the branch tip. We applied an automated algorithm to identify SYP puncta along the axon arbor and to measure their intensities to provide nonbiased quantitative data on SYP accumulation at individual presynaptic puncta (see Materials and Methods) (supplemental Fig. 1, available at www.jneurosci.org as supplemental material). In the example of short interval imaging of branch dynamics shown in Figure 2 (supplemental movie, available at www.jneurosci.org as supplemental material), only a minority of the terminal branches (32\%) observed were stable for the full $2 \mathrm{~h}$ of observation, but the SYP puncta on these stable branches were more intense $(61.8 \pm 5.2$ relative intensity units) than those found on transient branches with lifetimes shorter than $30 \mathrm{~min}(<20 \mathrm{~min}, 36.1 \pm 3.6$ relative intensity units, $p<0.01$; $\sim 20-29 \mathrm{~min}$, $37.4 \pm 4.0$ relative intensity units, $p<$ 0.05; Kruskal-Wallis with Dunn posttest). Branches with lifetimes that were $>30 \mathrm{~min}$ but shorter than the $2 \mathrm{~h}$ observation period had intermediate SYP punctum intensities (Fig. 2d). This result extends previous reports that branches bearing synaptic puncta are less likely to be eliminated than branches without puncta (Alsina et al., 2001; Hu et al., 2005) by demonstrating that even among branches with synapses, branch lifetimes in fact correlate directly with the degree of maturation of their presynaptic sites, as measured by the accumulation of SYP at puncta.

The presence of brighter presynaptic puncta on longer-lived branches could simply reflect the gradual accumulation of synaptic vesicles at presynaptic sites as they mature over time. However, another more intriguing possibility is that the maturation of synaptic sites might contribute directly to the stability of the branch on which the synapses reside. We therefore examined both of these possibilities by following the maturation and stability of individual SYP puncta in retinotectal axons over minutes to hours. 
SYP accumulates over time at newly formed synapses

New sites of SYP-CFP accumulation were consistently found to form just behind the advancing growth cones of actively elongating side branches. Gradual increases in fluorescence intensity at newly formed puncta were observed over the course of minutes (Fig. 3a). Our in vivo imaging protocol, which avoided extensive oversampling to reduce phototoxicity (see Materials and Methods), lacked the sensitivity to discern whether the accretion of SYPCFP at puncta occurred in discrete, quantized steps as has been reported for synaptogenesis in vitro (Ahmari et al., 2000; Bresler et al., 2004). Although evident within minutes, the increase in SYP punctum intensity continued for many hours after synapses first formed (Fig. $3 b)$. This progressive maturation of puncta on newly formed branches contrasts with the relatively stable punctum intensities on the main axon shaft, which showed small fluctuations but little net change in intensity over time (Fig. 2e).

We used our automated algorithm to quantify the fluorescence intensity changes of 1273 puncta from terminal branch tips on nine axons from nine tadpoles that had been imaged three times over $8 \mathrm{~h}$ to compare punctum lifetime with intensity. Puncta were inferred to have lifetimes of $<4 \mathrm{~h}$ when they were observed only in the third image and to have lifetimes from 4 to $8 \mathrm{~h}$ if they were observed in only the two final images. Fluorescence intensity increased significantly from puncta with lifetimes $<4 \mathrm{~h}(26.4 \pm 2.8)$ to puncta with lifetimes between 4 and $8 \mathrm{~h}(43.5 \pm 4.3$; $p<0.01$, ANOVA with Tukey-Kramer posttest) (Fig. 3c). However, even in puncta with lifetimes of $4-8 \mathrm{~h}$, punctum intensity has not reached fully mature levels. New synaptic vesicles continue to accumulate at presynaptic puncta over the subsequent hours causing an additional increase in mean fluorescence intensity to $68.1 \pm 3.5$ for puncta with lifetimes $>8 \mathrm{~h}(p<0.001$; ANOVA with TukeyKramer posttest).

These findings suggest that the amount of SYP present at an individual synaptic punctum, as measured by fluorescence intensity, provides an estimate of the relative age of that synapse. Indeed, the majority $(56 \pm 4.7 \%)$ of puncta with fluorescence intensity levels $<20 \mathrm{U}$ had formed recently (within the past $4 \mathrm{~h}$ ), whereas the fraction of newly formed puncta was significantly lower among synapses with higher levels of SYP accumulation (Fig. 3d) ( $p<0.01$; Kruskal-Wallis).

\section{Presynaptic sites with high SYP levels are more stable}

The fact that not all faint puncta are $<4$ h old, however, demonstrates that synapse maturation does not progress uniformly across all synapses. Time-lapse imaging clearly reveals that individual presynaptic puncta can either increase or decrease their relative intensities over time, and some puncta are eventually eliminated. As with the increase of SYP intensity at synaptic puncta over time, the loss of SYP labeling from synaptic sites is also a gradual rather than an abrupt process. On terminal branch tips, stable puncta that persisted for at least $8 \mathrm{~h}$ of imaging started with higher SYP intensities ( $63.2 \pm 0.7$ relative intensity units) in the first imaging time point than puncta that were lost $(28.0 \pm 0.5$ relative intensity units) over the following $8 \mathrm{~h}$ of imaging (supplemental Fig. 2, available at www.jneurosci.org as supplemental material) ( $p<0.001$, two-tailed $t$ test; $n=9$ axons). Only $10.6 \pm$ $2.9 \%$ of the brightest puncta ( $\geq 80$ relative intensity units) on branch tips were lost within $4 \mathrm{~h}$, whereas $37.9 \pm 4.5 \%$ of the faintest puncta $(<20 \mathrm{U})$ were eliminated over the same time period (Fig. 4) ( $p<0.001$; Kruskal-Wallis). Puncta of intermediate intensities were increasingly stable as labeling intensity increased. The prospective stability of presynaptic puncta is therefore directly correlated with their SYP intensities.

In vitro studies reveal that presynaptic sites accumulate proteins through the delivery of transport packets containing numerous presynaptic molecules (Zhai et al., 2001; Bresler et al., 2004). Although SYP has not been found in these transport packets, SYP-containing vesicles have also been shown in vitro to be actively transported through axons (Ahmari et al., 2000; Kaether et al., 2000). The imaging interval used in our in vivo experiments did not permit us to identify a rapidly transported pool of SYP in our axons. Although it is possible that a small fraction of the faintest puncta we observed may correspond to a labile, nonsynaptic pool of SYP, the conclusions drawn by comparing the relative stability of the faintest $(<20)$ and brightest $(>80)$ groups of SYP puncta are equally supported by comparing intermediate 


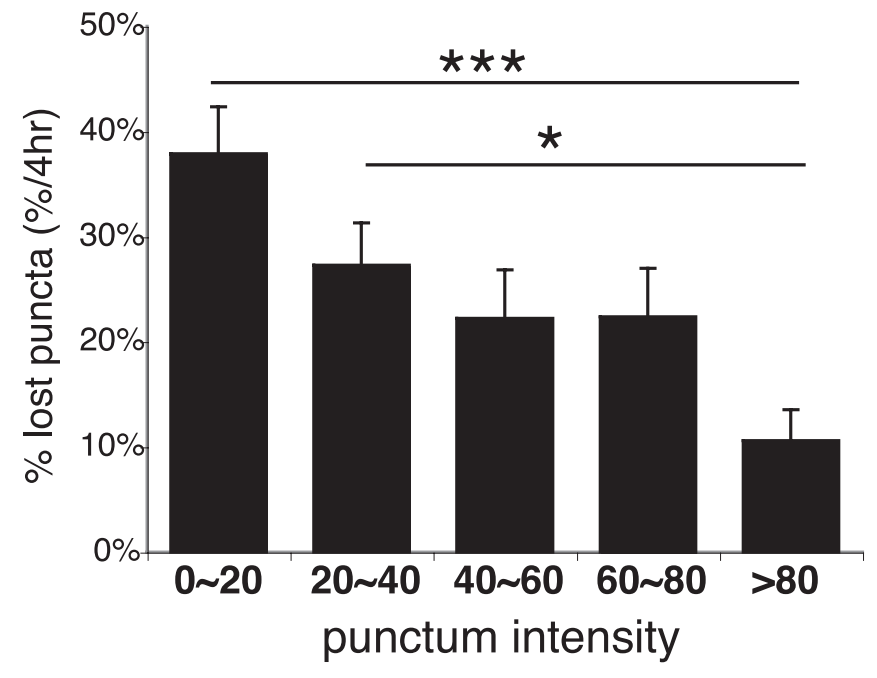

Figure 4. Stability of synaptic puncta correlates with the degree of synaptic vesicle accumulation. Punctum intensity is predictive of stability. The graph shows a fraction of puncta grouped by initial intensity that will be lost within the following $4 \mathrm{~h}$. ${ }^{* * *} p<0.001,{ }^{*} p<0.05$; KruskalWallis with Dunn posttest. Error bars indicate SE.

intensity puncta with the brightest group (Fig. 4). In addition, a sizable majority $(62 \%)$ of the initially faint puncta could still be detected after $4 \mathrm{~h}$, arguing against the possibility that most faint puncta are simply a labile, nonsynaptic SYP pool.

\section{Intense SYP puncta stabilize axon branches locally}

These data are consistent with a model in which transient branches can rapidly gain and lose synaptic sites, whereas more stable branches add new synapses that mature and accumulate more synaptic vesicles over time. However, this description does not differentiate between the incidental maturation of synapses that form on stable branches and the stabilization of branches as a consequence of their bearing mature synapses. To distinguish between these two mechanisms, we examined retracting branches to determine whether the presence of bright SYP puncta correlated with sites where branch retractions were stabilized.

Figure 5, $a$ and $b$, shows two examples of dynamic branches that partially retracted over $4 \mathrm{~h}$. Faintly labeled SYP puncta in the distal parts of the branches (open arrows) were eliminated as the branch retracted; however, in both cases, the branches stopped retracting at the very locations of the bright puncta, consistent with the idea that the branch is relatively more stable at these sites. We analyzed the locations of 502 SYP puncta from all of the retracting branches in a population of 16 time-lapse-imaged axon arbors from 15 animals kept in the dark to limit retinal activity to spontaneous levels during a $4 \mathrm{~h}$ interval between imaging sessions. In a scatterplot of the initial distances from the punctum to the branch tip (Fig. $5 c$, green lines, schematic; $d$, $x$-axis) versus the length that their branches retracted over a $4 \mathrm{~h}$ imaging interval (Fig. $5 c$, red line; $d, y$-axis), puncta that were eliminated by branch retraction fall to the right of the diagonal, puncta that were unaffected by the retraction are on the left side of the diagonal, and puncta that end up at the branch tip after retraction fall along the diagonal (retraction point). This graph clearly shows that very few intense SYP puncta are lost when branches retract and a large fraction of bright puncta is in the spared portion of the retracting branches. Furthermore, intensely labeled synaptic sites are concentrated within $1 \mu \mathrm{m}$ of the diagonal line representing the point at which retraction stopped (called the retraction point). The mean synaptic punctum intensity value for the portions of the branches that were retracted over $4 \mathrm{~h}$ (36.4 \pm 3.9$)$ was much lower than that measured at the point to which the branch had retracted after $4 \mathrm{~h}(61.9 \pm 6.3 ; p<0.05$, Kruskal-Wallis) (supplemental Fig. 3, available at www. jneurosci.org as supplemental material). A $\chi^{2}$ analysis of puncta in the 20-40 intensity range shows that their distribution between portions of the branches that were spared, lost, or at the retraction point was not significantly different from random $(p=0.41)$, whereas the analysis revealed a significantly higher number of brighter puncta (intensity values, $\geq 40$ ) at the retraction point than in the territory from which the branches had withdrawn $(p<0.05)$. If one considers the brightest puncta $(\geq 80)$, this correlation between the locations of bright puncta and the retraction point becomes even clearer $(p<0.0001)$ (Fig. $5 g$ ). Together, these data provide strong evidence that the point to which a branch retracts is at least in part determined by the presence of a bright SYP punctum at that point.

\section{Visual stimulation enhances stabilization of branches at bright puncta}

We have demonstrated previously that patterned visual stimulation increases the intrinsic excitability and the rate of dendritic growth of tectal neurons, the postsynaptic targets of RGC axons (Sin et al., 2002; Aizenman et al., 2003). The effects of visual stimulation on RGC axon arbor elaboration, however, remained unknown. To examine the effects of patterned visual stimulation on synaptic stabilization and growth of axon branches, RGC axons were imaged at three time points ( $n=10$ axons in 10 tadpoles). The first image was collected before any treatment. The second image was taken after the tadpole had been placed in darkness for $4 \mathrm{~h}$ to prevent visual stimulation. Animals were then exposed to $4 \mathrm{~h}$ of simulated motion visual stimulation, and a final image was collected (Sin et al., 2002).

As in unstimulated axons (Fig. $5 d$ ), branch retractions also halted at bright synaptic puncta during visual stimulation (Fig. $5 e$ ), and faint puncta were preferentially lost because of branch retractions. However, quantitative analysis revealed that intense presynaptic puncta were even more stable as a result of visual stimulation. The mean intensity of the SYP puncta that were lost through branch retraction during $4 \mathrm{~h}$ of visual stimulation $(24.6 \pm 3.2)$ was lower than that for puncta eliminated in the same axons during the preceding $4 \mathrm{~h}$ without visual stimulation $(35.8 \pm 6.3 ; p<0.01$, paired $t$ test) (Fig. $5 f)$. Visual stimulation did not result in a significant change in the average intensities of synaptic puncta in the spared parts of retracting branches (Fig. $5 f, g)$. However, the distribution of SYP puncta lost through branch retraction shifted with visual stimulation to include substantially fewer intense puncta than were lost during the unstimulated period (Fig. $5 g$ ). Therefore, by stabilizing bright puncta against elimination, visually driven activity was especially effective at restricting branch retraction to the parts of the branches that lacked dense accumulations of presynaptic vesicles.

\section{Effects of visual activity on RGC axon growth}

RGC axons in the optic tectum of tadpoles continually undergo extensive axon arbor remodeling. This is most evident in the exploratory behaviors of the distal branch tips of the axon arbor, whereas the more mature central core of the arbor is often remarkably stable over time (Fig. 6a). We investigated whether our visual stimulation paradigm, which enhanced the stability of bright puncta and had been previously shown to enhance dendritic outgrowth in tectal neurons, also had an effect on RGC axon branch dynamics and growth. Interestingly, we found that 
visual stimulation actually decreased the rate of axonal branch growth by decreasing the proportion of the branch tips that extend and increasing the fraction that retract (Fig. 6b,c). Together with the enhanced stabilization at bright SYP puncta seen in response to visual stimulation, this result suggests a model in which patterned visual activity refines the RGC arbor structure by causing the withdrawal of those exploratory branch tips that have not developed mature synapses, whereas concurrently enhancing the stability of the parts of the arbor that do bear mature synapses. This in turn would further bias the future addition of new branches to the locations of stable synaptic sites, leading to a compact, well-targeted arbor under conditions of visual stimulation.

\section{Discussion}

\section{Synaptic dynamics, axon arbor} structure, and topographic maps

Much of what is currently known about the dynamics of presynaptic terminal formation, maturation, and elimination during brain development comes from indirect measurements made by comparing electron micrographs of fixed anatomical material from different animals at different ages (Blue and Parnavelas, 1983; Vaughn, 1989) or from cultured neurons examined at various periods in vitro (for review, see Zhen and Jin, 2004; Ziv and Garner, 2004). Here, we used time-lapse imaging of fluorescently tagged SYP to follow the maturation of hundreds of individual presynaptic sites on growing RGC axons in the living brain and related synaptic dynamics to structural dynamics at the level of the entire axon arbor. We validated this method by showing that SYP clusters correspond to ultrastructurally identifiable presynaptic sites. The data reveal that individual retinal axons bear a variety of synapses ranging from sparse to dense accumulations of synaptic vesicles at any given moment, consistent with their ongoing structural rearrangement, and individual synapses can either accumulate SYP progressively as they mature or lose SYP as they disassemble. Previous studies from fixed tissue have reported that clusters of synaptic vesicle proteins form in axonal branches as quickly as $10 \mathrm{~min}$ after they form in vivo (Pinches and Cline, 1998). Our data confirm and extend this finding by demonstrating that these highly dynamic branches indeed contain SYP puncta, but that these puncta are measurably less intense than those in the stable skeleton of the arbor. Our observation that both maturation and disassembly of synapses in vivo are dynamic, gradual processes provides a simple mechanism for regulating the relative stability of synapses: as synaptic sites mature, they become increasingly resistant to elimination, because any
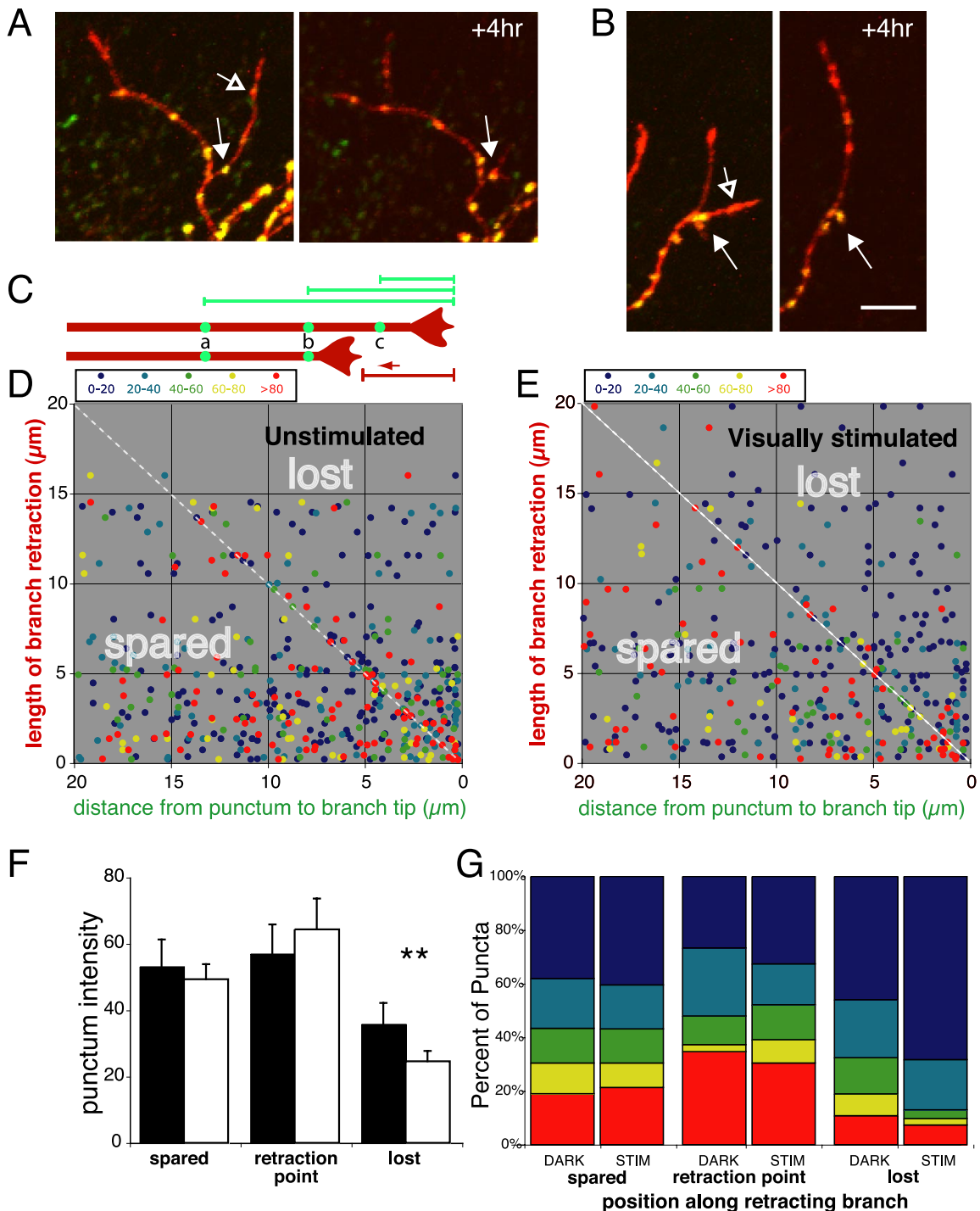

Figure 5. Branch retractions halt at bright SYP puncta: effects of visual stimulation. $\boldsymbol{A}, \boldsymbol{B}$, Examples of branch retractions over $4 \mathrm{~h}$ that stop at bright SYP puncta (closed arrow). Branches frequently retract past sites with faint puncta (open arrow). Scale bar, $10 \mu \mathrm{m}$. C, Schematic of measurements made on retracting branch tips. The bottom image represents the axon in the top image after undergoing retraction. Green lines are distances from puncta to the tips of their branches before retraction. The red line measures the distance retracted by the axon after $4 \mathrm{~h}$. Thus, puncta indicated by green circles are found in three different axonal territories: spared, the end point of retraction, or lost. D, Scatterplots of initial punctum locations along the branch versus branch length retracted made for puncta on all of the retracting branches from 16 axons imaged before and after $4 \mathrm{~h}$ of exposure to darkness. Punctum are color coded according to fluorescence intensities. Puncta on the right side of the diagonal line, indicating they were lost when the branch retracted, are less intense than those found within $1 \mu \mathrm{m}$ of the diagonal representing the point to which the branch retracted after $4 \mathrm{~h}$. $\boldsymbol{E}$, Same plot as $\boldsymbol{D}$ except tadpoles were exposed to $4 \mathrm{~h}$ of visual stimulation. $\boldsymbol{F}$, Intensity of puncta in the parts of axon branches that were spared, found within $1 \mu \mathrm{m}$ of the point to which the branch retracted (retraction point), or lost by the retraction in animals that were kept in darkness (black) or visually stimulated (white) during the $4 \mathrm{~h}$ imaging interval. ${ }^{* *} p<0.01 ; t$ test. Error bars indicate SE. G, Relative proportions of SYP puncta of various intensities (color scale as in D and $\boldsymbol{E}$ ) that started out in territory that was spared, within $1 \mu \mathrm{m}$ of the branch tip after retraction, or lost in the retraction.

stabilization of the contact that occurred must first be reversed before the synapse can be removed outright. Newer synapses are therefore less stable than older synaptic sites, but just as new synapses can either disassemble or mature over time, older synapses are similarly subject to either maintenance or destabilization and regression. Furthermore, we found that patterned visual activity enhances the stability of those synapses that have accumulated relatively more synaptic vesicles and the axonal arbor where they have formed while destabilizing parts of the arbor that lack these synaptic reinforcements. 


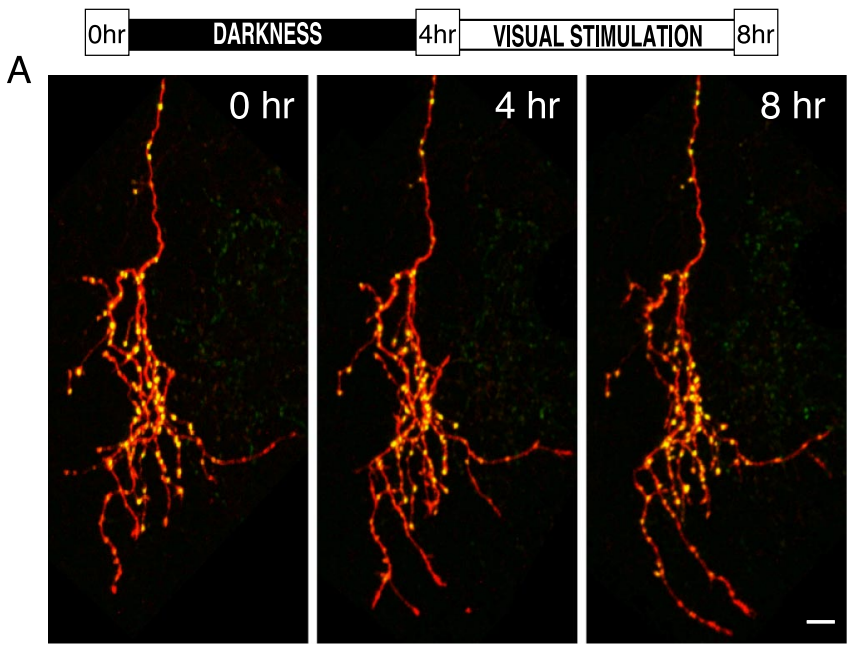

B
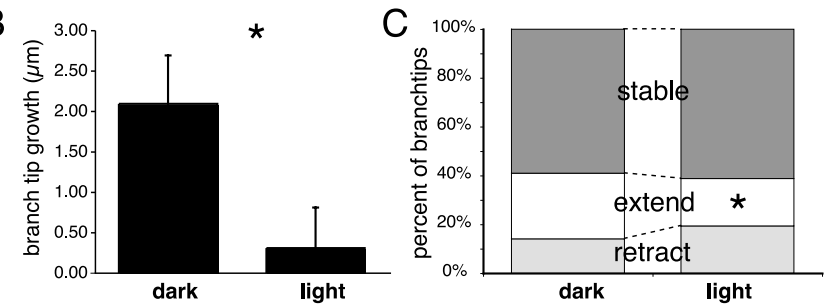

Figure 6. Visual stimulation consolidates the existing stable arbor and reduces exploratory growth. $\boldsymbol{A}$, SYP-GFP-expressing (green) and dsRED-MST-expressing (red) RGC axon arbor imaged in the optic tectum of a living tadpole at $4 \mathrm{~h}$ intervals, in darkness for the first $4 \mathrm{~h}$, and visually stimulated during the second $4 \mathrm{~h}$. The stable core of the arbor consists mainly of intense SYP puncta that can be followed over $8 \mathrm{~h}$, whereas the dynamic branch tips also bear many fainter, dynamic puncta. After visual stimulation, many branches are tipped by bright SYP puncta. $\boldsymbol{B}$, The mean increase in branch tip lengths is reduced by visual stimulation ( ${ }^{*} p<0.05$, paired $t$ test; $n=309$ light, 290 dark). C, Visual stimulation induces a shift in the distribution of branch tip behaviors with fewer branches extending and more retracting $\left({ }^{*} p<0.05 ; \chi^{2}\right.$ test). Stable branch tips are those that change by $<5$ $\mu \mathrm{m}$ in $4 \mathrm{~h}$. Scale bar, $10 \mu \mathrm{m}$.

This correlation between synaptic stability and branch dynamics has important implications for the overall structural dynamics of the axon arbor. As synaptic sites on branches mature, they may confer increased stability to the part of the branch on which they reside and may also serve as sites for further branch addition. These data suggest a model in which synaptogenesis and synapse maturation play important roles in topographic map refinement by sculpting the axonal projection at a local scale.

The retinotopic projection in frogs and fish remains plastic throughout life, because retinal axons continually shift their positions in the tectum to maintain retinotopy despite asymmetric patterns of neurogenesis in the retina and tectum (Easter and Stuermer, 1984; Reh and Constantine-Paton, 1984). In addition to continually updating the retinotopic map, retinotectal axons must also convey useful visual information to the brain of the tadpole. These two roles appear to be at odds with one another, the former requiring dynamic plasticity and the latter requiring predictable stability. It is therefore noteworthy that a substantial fraction of the retinotectal synapses, particularly the bright SYP puncta found in the stable skeleton of the axon arbor, showed little or no change over $8 \mathrm{~h}$ of imaging. The implication for retinotopic function is that the dynamic exploratory rearrangement of branches, including the making and breaking of new synapses, is likely to have a minimal impact on the net communication of visual information to tectal neurons over this time scale, but the impact of such rearrangements would increase over days and weeks as the population of stable synaptic sites gradually shifts (Cline, 1991; Ruthazer and Cline, 2004). Thus, the axonal arbor appears to have two different zones: a relatively stable zone that is important for maintaining a functional retinotopic projection and a dynamic zone that serves to test new connections for correlated patterns of afferent activity and further optimize the map. As the map shifts over many days and weeks, parts of the dynamic zone may be converted into the new stable zone, and the stable zone may be gradually disassembled, itself becoming dynamic.

\section{Mechanisms of structural plasticity}

Neural activity has been shown to regulate axonal arbor remodeling through a variety of mechanisms that control branch addition, elimination, and stabilization. Certain aspects of axon remodeling are believed to involve a retrograde signal generated by postsynaptic activation of NMDA-type glutamate receptors (Cline and Constantine-Paton, 1989; Rajan et al., 1999; Schmidt et al., 2000; Ruthazer et al., 2003) and their downstream signaling cascades (Zou and Cline, 1996; Schmidt et al., 2004). An NMDA receptor-dependent retrograde signal might directly act to induce the stabilization and maturation of the presynaptic terminal, thereby stabilizing its axonal branch and inducing further axonal branch elaboration near that site. Consistent with this idea, Hu et al. (2005) have reported that NMDA receptor blockade dispersed synaptobrevin-GFP clusters. Although we did not observe accelerated accumulation of SYP at synaptic puncta with visual stimulation, we did see evidence that vision increased branch stabilization at established synaptic sites. This suggests that beyond a certain threshold level of synaptic maturation, branch stabilization and further synapse maturation are separable phenomena and thus may rely on multiple interacting signaling mechanisms. For example, local branch stabilization could be mediated by transsynaptic cytoskeletal interactions via synaptic adhesion molecules such as cadherins (Yamagata et al., 2003). Synaptic maturation, in contrast, could be regulated through intracellular signaling of $\beta$-catenin, which both interacts extensively with the intracellular domain of cadherin at synapses (Uchida et al., 1996) and also has been found to be essential for the aggregation of synaptic vesicles at developing synapses (Bamji et al., 2003) or through diffusible molecules like BDNF (Hu et al., 2005) or bone morphogenetic proteins (McCabe et al., 2004).

Visual stimulation reduced exploratory peripheral branch elongation of retinotectal axons, whereas axons increased extensions with spontaneous levels of activity. This is in striking contrast to the growth-promoting effect on tectal neuron dendrites observed with the identical visual stimulation paradigm (Sin et al., 2002). In fact, there are fundamental differences in how axons and dendrites respond to changes in neural activity in vivo. Systemic blockade of action potential firing or of synaptic transmission through NMDA receptors causes a decrease in tectal neuron dendrite outgrowth but an increase in RGC axon branch dynamics (Rajan et al., 1999). The decrease in exploratory axon branch extension we observed with enhanced visual stimulation is consistent with these data. The opposite effects of activity blockade in axons and dendrites may be attributable to, in part, the differences in the axonal and dendritic cytoskeletal response to Rho GTPase activation (Ruchhoeft et al., 1999; Li et al., 2000), which is itself sensitive to neuronal activity (Li et al., 2002). Interestingly, when individual RGC axons are genetically silenced in an 
environment of active neighbors, they also exhibit less growth (Hua et al., 2005). This impairment is rescued by silencing neighboring RGC axons, suggesting that competitive interactions regulate axon growth. This raises the interesting possibility that visual stimulation regulates arbor growth and topographic map refinement through mechanisms based on competition for a limited resource. The consolidation of the axon arbor back to stable puncta during visual stimulation could therefore reflect the outcome of an activity-dependent competition with neighboring axons.

The functional accuracy of neuronal circuits depends directly on the precision of axonal inputs and the selection of appropriate synaptic contacts. Previous work has demonstrated that neural activity, and synaptic transmission in particular, can influence the morphogenesis of axon arbors in their termination areas (Schmidt, 2004). Such control of axon structural plasticity may be exerted at a very local level, including the regulation of the stability of individual branch tips (Ruthazer et al., 2003). This study now provides a mechanism by which axon branch stability is regulated at the scale of individual synapses. The stability of individual presynaptic sites increases as they gradually accumulate a large pool of synaptic vesicles, a classic morphological indicator of synaptic maturity. This finding suggests a model for axon branch remodeling in which axons extend exploratory side branches from a stable core arbor that make many immature synaptic contacts with postsynaptic target neurons. The majority of these contacts are lost over time, but a few succeed in maturing and become stable. These new stable synaptic sites in turn become part of the stable core arbor and serve as points for additional exploration by new branches. We found that visual stimulation, which causes accelerated growth of postsynaptic tectal dendrites, actually slows axonal growth by reducing exploratory branch extension, maintaining the arbor in a form defined by its stable core. This coordination of stable and dynamic zones within the axon arbor provides an ideal means for continuous remodeling and refinement of the arbor while preserving the functional utility of the neural circuit.

\section{References}

Ahmari SE, Buchanan J, Smith SJ (2000) Assembly of presynaptic active zones from cytoplasmic transport packets. Nat Neurosci 3:445-451.

Aizenman CD, Akerman CJ, Jensen KR, Cline HT (2003) Visually driven regulation of intrinsic neuronal excitability improves stimulus detection in vivo. Neuron 39:831-842.

Alsina B, Vu T, Cohen-Cory S (2001) Visualizing synapse formation in arborizing optic axons in vivo: dynamics and modulation by BDNF. Nat Neurosci 4:1093-1101.

Bamji SX, Shimazu K, Kimes N, Huelsken J, Birchmeier W, Lu B, Reichardt LF (2003) Role of beta-catenin in synaptic vesicle localization and presynaptic assembly. Neuron 40:719-731.

Bevis BJ, Glick BS (2002) Rapidly maturing variants of the Discosoma red fluorescent protein (DsRed). Nat Biotechnol 20:83-87.

Blue ME, Parnavelas JG (1983) The formation and maturation of synapses in the visual cortex of the rat. II. Quantitative analysis. J Neurocytol 12:697-712.

Bresler T, Shapira M, Boeckers T, Dresbach T, Futter M, Garner CC, Rosenblum K, Gundelfinger ED, Ziv NE (2004) Postsynaptic density assembly is fundamentally different from presynaptic active zone assembly. J Neurosci 24:1507-1520.

Cline HT (1991) Activity-dependent plasticity in the visual systems of frogs and fish. Trends Neurosci 14:104-111.

Cline HT, Constantine-Paton M (1989) NMDA receptor antagonists disrupt the retinotectal topographic map. Neuron 3:413-426.
Easter Jr SS, Stuermer CA (1984) An evaluation of the hypothesis of shifting terminals in goldfish optic tectum. J Neurosci 4:1052-1063.

Hu B, Nikolakopoulou AM, Cohen-Cory S (2005) BDNF stabilizes synapses and maintains the structural complexity of optic axons in vivo. Development 132:4285-4298.

Hua JY, Smear MC, Baier H, Smith SJ (2005) Regulation of axon growth in vivo by activity-based competition. Nature 434:1022-1026.

Javaherian A, Cline HT (2005) Coordinated motor neuron axon growth and neuromuscular synaptogenesis are promoted by CPG15 in vivo. Neuron 45:505-512.

Kaether C, Skehel P, Dotti CG (2000) Axonal membrane proteins are transported in distinct carriers: a two-color video microscopy study in cultured hippocampal neurons. Mol Biol Cell 11:1213-1224.

Kaethner RJ, Stuermer CA (1992) Dynamics of terminal arbor formation and target approach of retinotectal axons in living zebrafish embryos: a time-lapse study of single axons. J Neurosci 12:3257-3271.

Li Z, Van Aelst L, Cline HT (2000) Rho GTPases regulate distinct aspects of dendritic arbor growth in Xenopus central neurons in vivo. Nat Neurosci 3:217-225.

Li Z, Aizenman CD, Cline HT (2002) Regulation of rho GTPases by crosstalk and neuronal activity in vivo. Neuron 33:741-750.

McCabe BD, Hom S, Aberle H, Fetter RD, Marques G, Haerry TE, Wan H, O'Connor MB, Goodman CS, Haghighi AP (2004) Highwire regulates presynaptic BMP signaling essential for synaptic growth. Neuron 41:891-905.

McGee AW, Bredt DS (2003) Assembly and plasticity of the glutamatergic postsynaptic specialization. Curr Opin Neurobiol 13:111-118.

McLaughlin T, O'Leary DD (2005) Molecular gradients and development of retinotopic maps. Annu Rev Neurosci 28:327-355.

Nieuwkoop PD, Faber J (1956) Normal table of Xenopus laevis (Daudin); a systematical and chronological survey of the development from the fertilized egg till the end of metamorphosis. Amsterdam: North-Holland.

O'Rourke NA, Cline HT, Fraser SE (1994) Rapid remodeling of retinal arbors in the tectum with and without blockade of synaptic transmission. Neuron 12:921-934.

Panzer JA, Song Y, Balice-Gordon RJ (2006) In vivo imaging of preferential motor axon outgrowth to and synaptogenesis at prepatterned acetylcholine receptor clusters in embryonic zebrafish skeletal muscle. J Neurosci 26:934-947.

Pinches EM, Cline HT (1998) Distribution of synaptic vesicle proteins within single retinotectal axons of Xenopus tadpoles. J Neurobiol $35: 426-434$

Rajan I, Witte S, Cline HT (1999) NMDA receptor activity stabilizes presynaptic retinotectal axons and postsynaptic optic tectal cell dendrites in vivo. J Neurobiol 38:357-368.

Reh TA, Constantine-Paton M (1984) Retinal ganglion cell terminals change their projection sites during larval development of Rana pipiens. J Neurosci 4:442-457.

Ruchhoeft ML, Ohnuma S, McNeill L, Holt CE, Harris WA (1999) The neuronal architecture of Xenopus retinal ganglion cells is sculpted by rhofamily GTPases in vivo. J Neurosci 19:8454-8463.

Ruthazer ES, Cline HT (2004) Insights into activity-dependent map formation from the retinotectal system: a middle-of-the-brain perspective. J Neurobiol 59:134-146.

Ruthazer ES, Akerman CJ, Cline HT (2003) Control of axon branch dynamics by correlated activity in vivo. Science 301:66-70.

Schmidt JT (2004) Activity-driven sharpening of the retinotectal projection: the search for retrograde synaptic signaling pathways. J Neurobiol 59:114-133.

Schmidt JT, Buzzard M (1993) Activity-driven sharpening of the retinotectal projection in goldfish: development under stroboscopic illumination prevents sharpening. J Neurobiol 24:384-399.

Schmidt JT, Buzzard M, Borress R, Dhillon S (2000) MK801 increases retinotectal arbor size in developing zebrafish without affecting kinetics of branch elimination and addition. J Neurobiol 42:303-314.

Schmidt JT, Fleming MR, Leu B (2004) Presynaptic protein kinase C controls maturation and branch dynamics of developing retinotectal arbors: possible role in activity-driven sharpening. J Neurobiol 58: $328-340$. 
Shapira M, Zhai RG, Dresbach T, Bresler T, Torres VI, Gundelfinger ED, ZivNE, Garner CC (2003) Unitary assembly of presynaptic active zones from Piccolo-Bassoon transport vesicles. Neuron 38:237-252.

Sin WC, Haas K, Ruthazer ES, Cline HT (2002) Dendrite growth increased by visual activity requires NMDA receptor and Rho GTPases. Nature 419:475-480.

Sudhof TC (2000) The synaptic vesicle cycle revisited. Neuron 28:317-320.

Uchida N, Honjo Y, Johnson KR, Wheelock MJ, Takeichi M (1996) The catenin/cadherin adhesion system is localized in synaptic junctions bordering transmitter release zones. J Cell Biol 135:767-779.

Vaughn JE (1989) Fine structure of synaptogenesis in the vertebrate central nervous system. Synapse 3:255-285.

Witte S, Stier H, Cline HT (1996) In vivo observations of timecourse and distribution of morphological dynamics in Xenopus retinotectal axon arbors. J Neurobiol 31:219-234.

Yamagata M, Sanes JR, Weiner JA (2003) Synaptic adhesion molecules. Curr Opin Cell Biol 15:621-632.

Zhai RG, Vardinon-Friedman H, Cases-Langhoff C, Becker B, Gundelfinger ED, Ziv NE, Garner CC (2001) Assembling the presynaptic active zone: a characterization of an active one precursor vesicle. Neuron 29:131-143.

Zhen M, Jin Y (2004) Presynaptic terminal differentiation: transport and assembly. Curr Opin Neurobiol 14:280-287.

Ziv NE, Garner CC (2004) Cellular and molecular mechanisms of presynaptic assembly. Nat Rev Neurosci 5:385-399.

Zou DJ, Cline HT (1996) Control of retinotectal axon arbor growth by postsynaptic CaMKII. Prog Brain Res 108:303-312. 